\title{
Propriedades Químicas e Energéticas de Madeiras Amazônicas do Segundo Ciclo de Corte
}

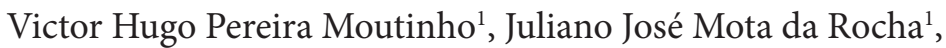 \\ Emanuelle Printes do Amaral ${ }^{1}$, Lucas Geovane de Medeiros Santana ${ }^{1}$, \\ Osmar José Romeiro de Águiar ${ }^{2}$
}

${ }^{1}$ Instituto de Biodiversidade e Florestas, Universidade Federal do Oeste do Pará - UFOPA, Santarém/PA, Brasil ${ }^{2}$ Centro de Pesquisa Aqropecuária do Trópico Úmido, Embrapa Amazônia Oriental, Belém/PA, Brasil

\begin{abstract}
RESUMO
O objetivo do estudo foi determinar as propriedades químicas e energéticas da madeira de urucu da mata (Bixa arborea), breu (Protium apiculatum) e acariquarana (Rinorea guianensis), para melhor aproveitamento dos resíduos gerados na obtenção de madeira serrada dessas espécies florestais. Foram coletadas 5 árvores de cada espécie e retirados discos à altura de 2 metros do solo, os quais foram transformados em cunhas para a obtenção de serragem para as análises químicas e energéticas. A avaliação dos resultados foi realizada pelo teste de comparação de médias de Tukey $(\mathrm{p} \leq 0,05)$. Bixa arborea foi a espécie que obteve maior poder calorífico superior e teor de lignina, todavia, menor densidade. Protiumapiculatum e Rinorea guianesis demonstraram ter características químicas e energéticas favoráveis à produção de carvão vegetal e de lenha para fins doméstico e em indústrias como olarias.
\end{abstract}

Palavras-chave: resíduos de serraria, bioenergia, Protium sp., Bixa sp., Rinorea sp.

\section{Chemical and Energetic Properties of Amazonian Woods of the Second Cutting Cycle}

\begin{abstract}
The aim of this study was to determine the chemical and energetic properties of Bixa arborea, Protium apiculatum, and Rinorea guianensis woods in order to support the recovery of residues in the lumber production of these species, also considering the lack of studies on their technological properties. For this purpose, five trees of each species were collected and, subsequently, discs were removed from the height of 2 meters above surface; they were processed in wedges to obtain sawdust for analysis. The evaluation of the results was performed by the Tukey's mean comparison test at $0.05 \%$ probability level. $B$. arborea was the species with the highest gross calorific value and lignin content, but lower specific gravity. P. apiculatum and R. guianesis showed chemical and energetic characteristics favorable to charcoal production and firewood.
\end{abstract}

Keywords: sawmill residue, bioenergy, Protium sp., Bixa sp., Rinorea sp. 


\section{INTRODUÇÃO}

Em 1979, uma área de 124 ha na Floresta Nacional do Tapajós foi submetida à colheita de árvores comerciais com intuito de avaliar os impactos pós exploração e monitorar a regeneração da floresta no local em que, ao longo dos últimos 30 anos, foram realizados estudos de inventário florestal para acompanhar o seu crescimento (Costa \& Aguiar, 1980). Após esse período de monitoramento, observou-se que outras espécies se destacaram em relação àquelas colhidas na década de 1970, porém não há estudos das propriedades tecnológicas da madeira de diversas delas, muito menos de seus resíduos.

Estima-se que do volume total de uma árvore sejam aproveitados em torno de $40 \%$ a $60 \%$ no processamento da tora, significando que a cada 10 árvores colhidas para produção de madeira serrada, apenas o volume referente a 5 são aproveitadas comercialmente (Gomes, 2004), o restante se transforma em resíduos que, em sua maioria, não são aproveitados, podendo se transformar em poluentes ambientais. Dentre as diferentes opções para o aproveitamento deles, pode-se citar a geração de energia pela queima direta ou a transformação dos resíduos em briquetes, pellets ou carvão vegetal.

Atualmente, tais resíduos estão deixando de ser um problema ambiental, haja vista que parte deles estão sendo utilizados como fonte de energia (Nascimento \& Biaggioni, 2010). Nesse sentido, nos últimos anos, a madeira tornou-se uma fonte energética favorável ambientalmente, por causa do balanço de carbono, em que tecnologias mais eficientes para a conversão da biomassa em energia térmica e elétrica têm sido promovidas, visando melhor aproveitamento da matéria-prima.

No Brasil, no ano de 2013, 41\% do consumo energético proveio de fontes renováveis, e a participação da lenha ou carvão vegetal foi de 9,5\% (EPE, 2014). A produção brasileira de carvão vegetal destina-se principalmente ao atendimento do setor industrial, como destaque para a siderurgia, além do consumo residencial.

Nesse sentido, a presença de alto teor de substâncias de natureza aromática, como extrativos e lignina na madeira, gera, como consequência, um carvão com maior densidade e mais resistente quanto a suas propriedades físico-mecânicas (Trugilho \& Silva, 2001).
O procedimento de transformação da madeira ou resíduo em carvão é conhecido como carbonização e consiste em concentrar carbono e expulsar oxigênio, com consequente aumento energético do produto final (Santos, 2008).

Diante do exposto, o trabalho objetivou determinar as propriedades químicas e energéticas da madeira de urucu da mata (Bixa arborea), breu (Protium apiculatum) e acariquarana (Rinorea guianensis), para melhor aproveitamento dos resíduos gerados na obtenção de madeira serrada dessas espécies florestais.

\section{MATERIAL E MÉTODOS}

O material foi coletado no decorrer de 23 a 25 de outubro de 2012, na Floresta Nacional do Tapajós FLONA, km 67 da Rodovia BR 163, coordenadas S2 ${ }^{\circ} 53^{\prime} 8^{\prime \prime}$ e W54 $55^{\prime} 16,7^{\prime \prime}$, na área experimental da Empresa Brasileira de Pesquisa Agropecuária - EMBRAPA Amazônia Oriental. As árvores foram selecionadas por meio dos critérios de abundância, frequência $e$ dominância na área de estudo, além da ausência de pesquisa na área de tecnologia de produtos florestais.

Foram colhidas aleatoriamente 5 árvores de cada espécie, sendo retirados discos na altura de 2 metros, onde, na ocasião, também se coletou material botânico, sendo este encaminhado ao herbário da Embrapa Amazônia Oriental no intuito de realizar a identificação das espécies florestais.

Para as análises químicas, os discos obtidos do tronco a 2 metros de altura foram transformados em maravalhas, em uma plaina, e, posteriormente, em serragem, em moinho de facas. O material obtido foi peneirado e utilizou-se a fração que ficou retida entre as peneiras de 40 e 60 mesh. As amostras foram armazenadas em frascos hermeticamente fechados e conduzidas para a sala de aclimatação, onde permaneceram até a estabilização de sua massa, para posterior obtenção da porcentagem de umidade absolutamente seca (\% AS) da mesma.

Para a determinação de extrativos em água quente, água fria e etanol-tolueno, adotou-se a norma da Associação Brasileira Técnica de Celulose e Papel ABTCP (1974). Já o teor de lignina insolúvel foi obtido de acordo com a metodologia proposta por Gomide \& Demuner (1986). 
Para a análise imediata do carvão vegetal adotou-se a norma da American Society for Testing and Materials - ASTM D-7582 (ASTM, 2010). Para o poder calorífico, utilizou-se uma bomba calorimétrica, seguindo-se as informações contidas na NBR 8633, ABNT (1983).

Para o processo de carbonização, as cunhas obtidas dos discos de madeira foram a forno mufla com temperatura máxima de carbonização de $450^{\circ} \mathrm{C}$ e taxa de aquecimento média de $1^{\circ} \mathrm{C} \mathrm{min}{ }^{-1}$, durante $1 \mathrm{~h}$, após a estabilização da temperatura máxima. Os rendimentos gravimétricos do carvão vegetal, assim como as perdas volumétricas inerentes à degradação térmica da madeira foram determinados relacionando-os com a massa das cunhas absolutamente secas.

$\mathrm{Na}$ avaliação dos dados utilizou-se delineamento inteiramente casualizado com 3 repetições por tratamento, para os tratamentos que apresentaram diferenças significativas pelo teste $\mathrm{F}$ ( $\mathrm{p} \leq 0,05)$, as médias foram comparadas pelo teste de Tukey $(\mathrm{p} \leq 0,05)$.

\section{RESULTADOS E DISCUSSÃO}

Os resultados obtidos na análise de extrativos evidenciaram que houve diferença significativa entre as três espécies: Rinorea guianensis obteve os maiores valores dentre as estudadas, enquanto Protium apiculatum e Bixa arborea apresentaram menores médias (Tabela 1).

Para Jankowsky \& Galvão (1979), os extrativos solúveis em água provenientes de folhosas possuem correlação negativa com a umidade de equilíbrio do material e causam redução da higroscopicidade o que, segundo Pereira (2012), pode, inclusive, dificultar a perda de umidade no decorrer da carbonização, isso porque a retirada de água da parte mais interna para a parte mais externa da madeira fica dificultada (Galvão \& Jankowsky, 1985) influenciando negativamente, em consequência, as propriedades de resistência e densidade do resíduo carbonífero (Moutinho, 2011).

Moutinho et al. (2011), ao estudarem 6 espécies amazônicas do gênero Eschewilera Mart Ex DC, encontraram valores de $3,6 \%$ a $11,1 \%$ de extrativos totais, corroborando resultados obtidos para as espécies estudadas.

Segundo Bufalino et al. (2012) e Castro et al. (2013), a presença de elevados teores de extrativos é uma característica vantajosa para a produção de energia, por causa do poder calorífico desses componentes. Para Medeiros et al. (2014), a presença de extrativos na madeira é um ponto importante quando se almeja produzir carvão vegetal, pois eles aumentam a densidade da madeira, favorecendo assim a produção de um carvão mais denso.

Quanto ao teor de lignina, não houve diferença estatística entre as espécies. Trugilho et al. (1991) e Moutinho et al. (2011), ao estudarem espécies nativas da Amazônia, encontraram médias variando entre $23 \%$ e $29 \%$ de lignina, enquanto Silva et al. (2014), ao avaliarem as propriedades energéticas dos resíduos de madeiras tropicais, encontraram média de 27,9\%.

Nisso, ressalta-se que Trugilho et al. (2001), ao trabalharem com clones de Eucalyptus, principal espécie madeireira utilizada na produção de energia no país, encontraram teor de lignina variando de $27 \%$ a $32 \%$. Queiroz \& Barrichelo (2008) e Protásio et al. (2014) alertam que as espécies de Eucalyptus passaram por anos de melhoramento visando, entre outros objetivos, aumentar seu teor de lignina, ratificando assim o potencial das espécies nativas em estudo para produção de energia e melhoramento genético em decorrência do alto teor de lignina encontrado.

Ressalta-se que a lignina é um dos componentes mais importantes na conversão da madeira em carvão,

Tabela 1. Teores de extrativos, de lignina e de holocelulose das espécies florestais estudadas.

Table 1. Extractive, lignin and holocellulose contents of studied forestry species.

\begin{tabular}{|c|c|c|c|c|c|}
\hline \multirow{2}{*}{$\begin{array}{l}\text { Espécies } \\
\text { estudadas }\end{array}$} & \multicolumn{3}{|c|}{ Extrativos (\%) } & \multirow{2}{*}{ Lignina } & \multirow{2}{*}{ Holocelulose } \\
\hline & Água fria & Água quente & Totais & & \\
\hline B. arborea & $0,71 \mathrm{a}$ & $1,90 \mathrm{a}$ & $3,81 \mathrm{a}$ & $29,58 \mathrm{a}$ & $63,99 \mathrm{a}$ \\
\hline P. apiculatum & $1,36 \mathrm{~b}$ & $3,13 \mathrm{~b}$ & $4,64 \mathrm{~b}$ & $26,57 \mathrm{a}$ & $64,30 \mathrm{a}$ \\
\hline R. guianesis & $2,50 \mathrm{c}$ & $3,39 c$ & $6,10 \mathrm{c}$ & $27,69 \mathrm{a}$ & $60,33 \mathrm{~b}$ \\
\hline
\end{tabular}

Médias seguidas de mesma letra não diferem entre si segundo o teste de Tukey ( $\mathrm{p}>0,05 \%)$. 
de forma que seu teor e tipo são parâmetros importantes pois, em geral, espera-se que essa conversão aumente com a proporção de lignina total e a diminuição da relação siringila:guaiacila da lignina, uma vez que isso promoveria maior resistência a degradação térmica devido a presença de estruturas mais condensadas (Castro et al., 2013). Brito \& Barrichelo (1977) e Oliveira et al. (1982) constataram que embora a lignina comece a se degradar sob o efeito de temperaturas relativamente baixas, em torno de $150{ }^{\circ} \mathrm{C}$, observa-se que, ao contrário da celulose e das hemiceluloses, sua decomposição é mais lenta, apresentando maior resistência à degradação térmica.

Para holocelulose houve diferença estatística segundo o teste de Tukey a $5 \%$ de probabilidade, no qual $R$. guianenses obteve menor média, sendo que $B$. arborea e P. apiculatum apresentaram médias próximas de $65 \%$. A presença de holocelulose em grandes quantidades é indesejável quando se objetiva a produção de carvão vegetal, por causa de sua maior instabilidade térmica (Costa et al., 2014; Gani \& Naruse, 2007).

Houve diferença estatística para o poder calorífico superior (Tabela 2) e B. arborea foi a espécie que obteve maior média entre as espécies estudadas; P. apiculatum e R. guianesis não apresentaram diferenças de média segundo o teste de Tukey.

Segundo Jara (1989), o poder calorífico da madeira pode ser influenciado pela umidade e por sua constituição química, principalmente lignina e extrativos, por esses possuírem menos oxigênio em sua composição, quando comparados aos polissacarídeos presentes na holocelulose, os quais apresentam estruturas pouco complexas e um número menor de ligação C-C, tendo dessa forma maior tendência à degradação térmica (Silva et al., 2014).

O valor obtido para o teor de cinzas foi variável entre as espécies deste estudo, sendo que P. apiculatum apresentou maior média, seguida de $R$. guianesis e B. arborea. Medeiros et al. (2014) relatam que teores de cinzas elevados na madeira podem provocar danos em fornalhas e em outros equipamentos em que ela é utilizada como fonte de energia.

Em paralelo, Oliveira (2003) informou que quanto maior a proporção de minerais na madeira, maior será o teor de cinzas no carvão. De acordo com Sturion et al. (1988), o teor de cinzas é fração mineral existente na madeira que permanece como resíduo após a combustão do carvão vegetal, variando de $0,5 \%$ a mais de $5 \%$. Esse é um dos fatores que contribuem para a indicação de P. apiculatum e R. guianensis na produção de energia pois, segundo Rocha (2011), são desejáveis baixos valores, já que os óxidos minerais formam incrustações nos equipamentos e nas tubulações quando queimados em fornalhas, além de reduzirem a inflamabilidade do combustível.

Para os resultados da densidade básica, houve diferença significativa a $5 \%$ de probabilidade entre as espécies estudadas, de forma que $R$. guianensis obteve maior média, de $0,98 \mathrm{~g} . \mathrm{cm}^{-3}$, sendo considerada madeira de alta densidade, segundo a classificação de Melo et al. (1990), seguida de P. apiculatum e B. Arborea, com densidades consideradas média e baixa, respectivamente.

Segundo Brito \& Barrichelo (1977), Oliveira et al. (2010) e Moutinho (2011), a densidade da madeira possui relação direta com a densidade do carvão. Tal fato torna-a um dos parâmetros para a seleção de espécies na produção de energia (Silva et al., 2007). Porém a espécie $B$. Arbórea, por ter apresentado baixa densidade, possivelmente terá como produto carvão de baixa densidade energética.

Entretanto, Quirino et al. (2012) sugerem que materiais de baixa densidade podem ser utilizados para geração de energia pelo processo de briquetagem, que consiste em compactar e homogeneizar o material, aumentando assim a densidade energética. Santos et al. (2011), ao avaliarem o potencial de briquetagem de resíduos florestais, notaram que, em termos de aumento de densidade dos briquetes, o processo gera melhores resultados para espécies com menor densidade básica, caso de B. arborea.

Para o rendimento de carvão, verificou-se que não houve diferenças estatísticas, tendo as espécies apresentado rendimento médio próximo a $40 \%$, em que B. arborea, apesar de possuir menor densidade dentre as espécies estudadas, apresentou maior valor absoluto de rendimento gravimétrico (Tabela 3). Nesse caso, citam-se os trabalhos de Brito \& Barrichelo (1977) e de Vale et al. (2001), os quais não encontraram correlação significativa entre densidade da madeira e rendimento em carvão vegetal.

Reis et al. (2012) e Brito et al. (1983), ao estudarem diferentes espécies do gênero Eucalyptus, encontraram 
Tabela 2. Teor de cinzas, poder calorífico superior e densidade aparente das espécies estudadas.

Table 2. Ash contents and superior calorific value of studied species.

\begin{tabular}{|c|c|c|c|}
\hline Espécies estudadas & $\begin{array}{l}\text { Poder calorífico superior } \\
\left(\mathrm{kcal} . \mathrm{kg}^{1}\right)\end{array}$ & Teor de cinzas (\%) & $\begin{array}{c}\text { Densidade básica } \\
\left(\mathrm{g} . \mathrm{cm}^{-3}\right)\end{array}$ \\
\hline B. arborea & $4794 \mathrm{a}$ & $0,12 \mathrm{a}$ & $0,33 \mathrm{c}$ \\
\hline P. apiculatum & $4615 b$ & $1,11 \mathrm{~b}$ & $0,60 \mathrm{~b}$ \\
\hline R. guianensis & $4665 \mathrm{~b}$ & $0,74 \mathrm{~b}$ & $0,98 \mathrm{a}$ \\
\hline
\end{tabular}

Médias seguidas de mesma letra não diferem entre si segundo o teste de Tukey ( $p>0,05 \%)$.

Tabela 3. Rendimentos gravimétricos em carvão vegetal, gases condensáveis e não condensáveis para as espécies estudadas.

Table 3. Gravimetric yield in charcoal, condensable gases and non-condensable gases to the studied species.

\begin{tabular}{lccc}
\multicolumn{1}{c}{$\begin{array}{c}\text { Espécies } \\
\text { estudadas }\end{array}$} & \multicolumn{3}{c}{ Rendimento (\%) } \\
\cline { 2 - 4 } \multicolumn{1}{c}{ B. arborea } & Carvão & Gases condensáveis & Gases não condensáveis \\
P. apiculatum & $40,16 \mathrm{a}$ & $30,53 \mathrm{~b}$ & $29,31 \mathrm{a}$ \\
R. guianensis & $39,59 \mathrm{a}$ & $32,17 \mathrm{~b}$ & $28,24 \mathrm{a}$ \\
\hline
\end{tabular}

Médias seguidas de mesma letra não diferem entre si segundo o teste de Tukey ( $\mathrm{p}>0,05 \%)$.

rendimentos gravimétricos entre $36,6 \%$ e $37,8 \%$, em condições metodológicas semelhantes, já Paes et al. (2012), ao estudarem o rendimento do carvão vegetal de 3 espécies de ocorrência no semiárido brasileiro, encontraram média de 40,39\%.

Para Protásio et al. (2011), o rendimento gravimétrico do carvão vegetal em proporções elevadas viabiliza maior aproveitamento de madeira e do espaço dos fornos durante o processo de carbonização. Quanto ao rendimento de gases condensáveis, $R$. guanensis obteve maior porcentagem, enquanto $B$. arborea e P. Altsonii não apresentaram diferenças estatísticas. Contudo, no processo de carbonização busca-se um menor rendimento em gases condensáveis, uma vez que, na maioria dos casos, eles não são recuperados (Paes et al., 2012).

Assis et al. (2012), ao estudarem clones híbridos de Eucalyptus grandis e Eucalyptus urophylla, encontraram valor médio de gases não condensáveis de 28,89\%. Além de substâncias voláteis que são responsáveis pela formação de chamas durante a combustão, esses gases são formados majoritariamente pela decomposição térmica da celulose e hemicelulose no decorrer do processo de carbonização (Martins, 1980). O efeito dessas ocorre na modificação estrutural do carvão, na porosidade e na massa específica, as quais podem ser afetadas no decorrer da sua eliminação (Barcelos, 2007).

\section{CONCLUSÃO}

Os resíduos madeireiros das espécies em estudos apresentam viabilidade potencial para utilização energética, seja na forma direta, seja transformados em adensados.

\section{STATUS DA SUBMISSÃO}

Recebido: 4 fev., 2015

Aceito: 14 mar., 2016

\section{AUTOR(ES) PARA CORRESPONDÊNCIA}

\section{Victor Hugo Pereira Moutinho}

Laboratório de Tecnologia da Madeira LTM, Instituto de Biodiversidade e Florestas, Universidade Federal do Oeste do Pará UFOPA, Rua Vera Paz, s/n, CEP 68035-110, Santarém, PA, Brasil

e-mail: victor.moutinho@ufopa.edu.br

\section{REFERÊNCIAS}

American Society for Testing and Material - ASTM. Standard test methods for proximate analysis of coal and coke by macro thermogravimetric analysis. West Conshohocken: ASTM; 2010. 
Assis MR, Protásio T, Assis CO, Trugilho PF, Santana WMS. Qualidade e rendimento do carvão vegetal de um clone híbrido de Eucalyptus grandis x Eucalyptus urophylla. Pesquisa Florestal Brasileira 2012; 32(71): 291-302. http://dx.doi.org/10.4336/2012.pfb.32.71.291.

Associação Brasileira de Normas Técnicas - ABNT. NBR 8633: carvão vegetal: determinação do poder calorífico: método de ensaio. Rio de Janeiro: ABNT; 1983.

Associação Brasileira Técnica de Celulose e Papel - ABTCP. Normas técnicas ABCTP. São Paulo: ABTCP; 1974.

Barcelos DC. Caracterização do carvão vegetal através do uso de espectroscopia no infravermelho próximo [tese]. Viçosa: Universidade Federal de Viçosa; 2007.

Brito JO, Barrichelo LEG, Seixas F, Migliorini AJ, Muramoto MC. Análise da produção energética e de carvão vegetal de espécies de eucalipto. Scientia Forestalis 1983; (23): 53-56.

Brito JO, Barrichelo LEG. Correlações entre características físicas e químicas da madeira e a produção de carvão vegetal: i. densidade e teor de lignina da madeira de Eucalipto. Scientia Forestalis 1977; (14): 9-20.

Bufalino L, Protásio TP, Couto AM, Nassur OAC, Sá VA, Trugilho PF et al. Caracterização química e energética para aproveitamento da madeira de costaneira e desbaste de cedro australiano. Brazilian Journal of Forestry Research 2012; 32(70): 129-137.

Castro AFNM, Castro RVO, Carneiro ACO, Lima JE, Santos RC, Pereira BLC et al. Análise multivariada para seleção de clones de eucalipto destinados à produção de carvão vegetal. Pesquisa Agropecuaria Brasileira 2013; 48(6): 627-635. http://dx.doi.org/10.1590/S0100204X2013000600008.

Costa PPC Fo, Aguiar OR. Exploração mecanizada da floresta tropical úmida sem babaçu. Belém: EMBRAPA; 1980. Circular técnica n. 9.

Costa TG, Bianchi ML, Protásio TB, Trugilho PF, Pereira A Jr. Qualidade da madeira de cinco espécies de ocorrência no cerrado para produção de carvão vegetal. Cerne 2014; 20(1): 37-46. http://dx.doi.org/10.1590/ S0104-77602014000100005.

Empresa de Pesquisas Energéticas - EPE. Balanço energético anual 2014: ano base 2013. Rio de Janeiro: EPE; 2014. 23 p.

Galvão APM, Jankowsky IP. Secagem racional da Madeira. São Paulo: Nobel; 1985. 112 p., vol. 1.

Gani A, Naruse I. Efeito do conteúdo de celulose e lignina sobre as características de pirólise e de combustão para diversos tipos de biomassa. Renewable Energy 2007; 32(4): 649-661. http://dx.doi.org/10.1016/j.renene.2006.02.017.

Gomes JI. Aproveitamento de resíduos de madeira em três empresas madeireiras do estado do Pará. Belém: EMBRAPA; 2004. Comunicado técnico.
Gomide JL, Demuner BJ. Determinação do teor de lignina em material lenhoso: método Klason modificado. O Papel 1986; 47(8): 36-8.

Jankowsky IP, Galvão APM. Influência do teor de extrativos na umidade de equilíbrio da madeira. Scientia Forestalis 1979; (18): 1-33.

Jara ERP. O poder calorífico de algumas madeiras que ocorrem no Brasil. São Paulo: Instituto de Pesquisas Tecnológicas; 1989. Comunicação Técnica n. 1797.

Martins H. Madeira como fonte de energia. In: Penedo WR. Uso da madeira para fins energéticos. Belo Horizonte: CETEC; 1980. p. 11-26.

Medeiros PN No, Oliveira L, Paes JB. Relações entre as características da madeira e do carvão vegetal de duas Espécies da Caatinga. Floresta e Ambiente 2014; 21(4): 484-493. http://dx.doi.org/10.1590/2179-8087.051313.

Melo JE, Coradin VTR, Mendes JC. Classes de densidade para madeiras da Amazônia brasileira. In: Anais do $6^{\circ}$ Congresso Florestal Brasileiro; 1990; Campos do Jordão. São Paulo: Sociedade Brasileira de Silvicultura; 1990. vol. 3, p. 695-699.

Moutinho VHP, Couto AM, Lima JT, Aguiar OJR, Nogueira MOG. Caracterização das madeiras conhecidas na Amazônia brasileira como mata-mata (Lethicidaceae fam, A. Rich). Science Forum 2011; 39(92): 457-461.

Moutinho, VHP. Influência da variabilidade dimensional e da densidade da madeira de Eucalyptus sp. e Corymbia sp. na qualidade do carvão [tese]. Piracicaba: Universidade de São Paulo; 2011.

Nascimento MD, Biaggioni MAM. Avaliação energética do uso da lenha e cavaco de madeira para produção de energia em agroindústria. Revista Energia na Agricultura 2010; 25(3): 104-117. http://dx.doi.org/10.17224/ EnergAgric.2010v25n3p104-117.

Oliveira CO, Carneiro ACO, Vital BR, Almeida W, Pereira BLC, Cardoso MT. Parâmetros de qualidade da madeira e do carvão vegetal de Eucalyptus pellita F. Muell. ScientiaForestalis 2010; 38(87): 431-439.

Oliveira E. Características anatômicas, químicas e térmicas da madeira de três espécies de maior ocorrência no SemiÁrido Nordestino [dissertação]. Viçosa: Universidade Federal de Viçosa; 2003.

Oliveira JB, Vivacqua A Fo, Mendes MG, Gomes PA. Produção de carvão vegetal: aspectos técnicos. In: Penedo WR, organizadores. Produção e utilização de carvão vegetal. Belo Horizonte: CETEC; 1982. p. 27-41.

Paes JB, Lima CR, Oliveira E, Santos HCM. Rendimento e caracterização do carvão vegetal de três Espécies de ocorrência no semiárido brasileiro. Ciência da Madeira 2012; 3(1): 1-10. http://dx.doi.org/10.12953/2177-6830. v03n01a01. 
Pereira BLC. Qualidade da madeira de Eucalyptus para a produção de carvão vegetal [dissertação]. Viçosa: Universidade Federal de Viçosa; 2012.

Protásio TP, Goulart SL, Neves TA, Trugilho PF, Ramalho FMG, Queiroz LMRSB. Qualidade da madeira e do carvão vegetal oriundos de floresta plantada em Minas Gerais. Brazilian Journal 2014; 34(78): 111-123.

Protásio TP, Santana JDP, Guimarães RM No, Guimarães JB Jr, Trugilho PF et al. Avaliação da qualidade do carvão vegetal de Qualea parviflora. Pesquisa Florestal Brasileira, Colombo 2011; 31(68): 295-307. http://dx.doi. org/10.4336/2011.pfb.31.68.295.

Queiroz LBS, Barrichelo LEG. Produtividade decorre da pesquisa coletiva. In: Queiroz LBS, Barrichelo LEG. A Celulose de Eucalipto uma oportunidade brasileira. Vinhedo: Avis Brasilis Editora; 2008

Quirino WF, Pinha IVO, Moreira ACO, Souza FMT Fo. Densitometria de raio $\mathrm{X}$ na análise da qualidade de briquetes de resíduos de madeira. Scientia Forestalis 2012; 40(96): 525-536.

Reis AP, Melo ICNA, Protásio TP, Trugilho PF, Carneiro ACO. O efeito de local e espaçamento na qualidade do carvão vegetal de um clone de Eucalyptus urophylla S. T. Blake. Floresta e Ambiente 2012; 19(4): 497-505. http:// dx.doi.org/10.4322/floram.2012.055.

Rocha MFV. Influência do espaçamento e da idade na produtividade e propriedades da madeira de Eucalyptus grandis $x$ Eucalyptus camaldulensis para energia [dissertação]. Viçosa: Universidade Federal de Viçosa; 2011.

Santos ID. Influência dos teores de lignina, holocelulose, extrativos na densidade básica e contração da madeira e nos rendimentos e densidade do carvão vegetal de cinco espécies lenhosas do cerrado [dissertação]. Brasília: Universidade de Brasília; 2008.
Santos RC, Carneiro ACO, Castro RVO, Pimenta AS, Castro AFNM, Marinho IV, Boas MAV. Potencial de briquetagem de resíduos florestais da região do Seriadó, no Rio Grande do Norte. Pesquisa Florestal Brasileira 2011; 31(68): 285-294.

Silva DA, Almeida VC, Viana LC, Klock U, Muniz GIB. Avaliação das propriedades energéticas de resíduos de madeiras tropicais com uso da espectroscopia NIR. Floresta e Ambiente 2014; 21(4): 561-568. http://dx.doi. org/10.1590/2179-8087.043414.

Silva MG, Numazawa S, Araujo MM, Nagaishi TYR, Galvão GR. Carvão de resíduos de indústria madeireira de três espécies florestais exploradas no município de Paragominas, PA. Acta Amazonica 2007; 37(1): 61-70. http://dx.doi.org/10.1590/S0044-59672007000100007.

Sturion, JA; Pereira, JCD; Chemin, MS. Qualidade da madeira de Eucalyptus vimanalis para fins energéticos em função do espaçamento e idade de corte. Colombo 1988; (16): 55-59.

Trugilho PF, Lima JT, Mori AF, Lino AL. Avaliação de clones de eucalyptus para produção de carvão vegetal. Cerne 2001; 7(2): 104-114.

Trugilho PF, Silva DA. Influência da temperatura final de carbonização nas características físicas e químicas do carvão vegetal de jatobá (Hymenea courbaril L.). Scientia Agrária 2001; 2(1-2): 45-53.

Trugilho PF, Silva DG, Frazão JL, Regazzi AJ. Caracterização de espécies nativas e exótica da Amazônia e do carvão vegetal. Revista Árvore 1991; 15(2): 144-151.

Vale AT, Costa AF, Gonçalez JC, Nogueira M. Relações entre a densidade básica da madeira, o rendimento e a qualidade do carvão vegetal de espécies do cerrado. Revista Árvore 2001; 25(89): 89-95. 